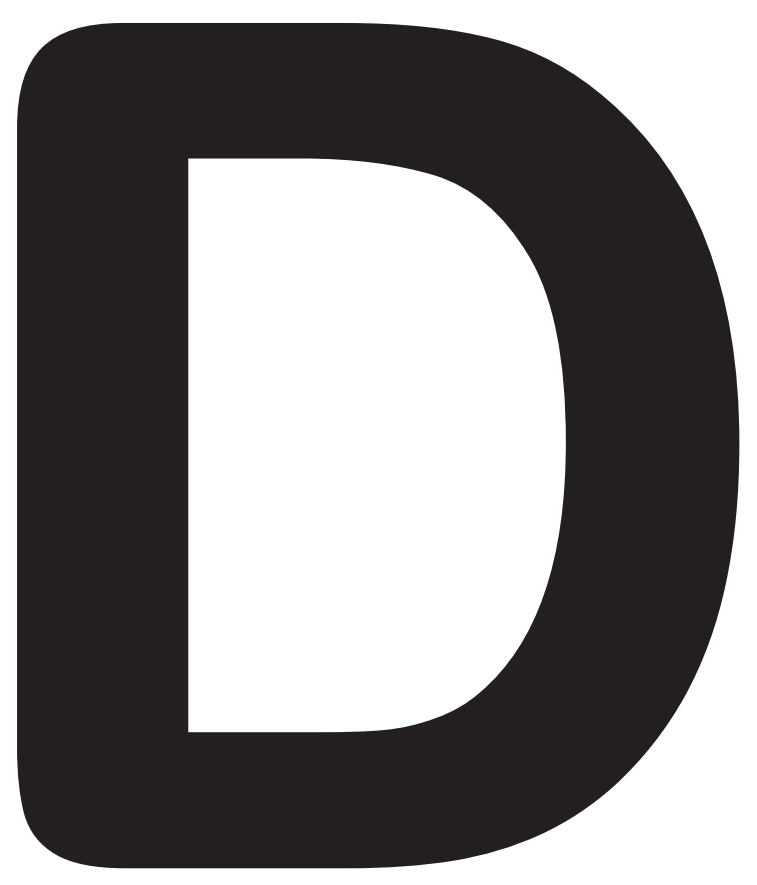

\title{
Satellite Symposia
}




\section{Friday, March 5}

\section{organised by GE Healthcare}

\section{SY 1}

Latest innovations in CT and MR: Improving patient care

Programme not available by date of print

$12: 30-13: 30$

Room E1

\section{organised by Bayer Schering Pharma}

\section{SY 2}

\section{Investigating: Excellent contrast in MRI}

Moderator:

V. Runge; Temple, TX/US

\section{$D-01$}

Welcome and introduction

V. Runge; Temple, TX/US

\section{D-02}

Investigating the CNS: Excellent contrast meets function M. Essig; Heidelberg/DE

Due to its high tissue contrast and its non-invasiveness, magnetic resonance (MR) imaging is accepted as the most sensitive method for imaging CNS diseases. In most diseases like tumour, infection, but even vascular diseases the use of paramagnetic contrast agents is mandatory. The selection of the optimal contrast agent, however, is crucial, taking into account the safety and contrasting behaviour of the available compounds. In the past few years a number of advanced, non enhanced and contrast enhanced MR imaging techniques have been developed that provide new insights into the pathophysiology of CNS diseases and proved to have a high impact on the disease management. These techniques include MR-spectroscopy, perfusion MR imaging, diffusion tensor MRI, dynamic contrast enhanced MRI and dynamic MRA studies. In this presentation the optimal use of gadobutrol (Gadovist $\otimes_{\text {, }}$ Bayer Healthcare, Berlin, Germany) in both conventional and functional imaging will be discussed using data from several ongoing and already completed study protocols on brain tumours, multiple sclerosis and cerebrovascular diseases. The authors will discuss the impact of an optimized contrast protocol on conventional and functional imaging techniques as well as MR field specific properties. Due to its high relaxivity both at low and high field, gadobutrol has shown a great performance in conventional MRI, e.g. in the assessment of brain tumors and multiple sclerosis. The high gadolinium concentration allows for a short and compact contrast media bolus which proved to be advantageous in dynamic MRI and MRA studies as well as in perfusion MRI.

Learning Objectives:

1. To appreciate the excellent contrast properties of gadobutrol in CNS diseases.

2. To become familiar with the use of functional imaging techniques in CNS diseases.

3. To learn about the optimal use of contrast media in functional MRI techniques.

\section{D-03}

Investigating MRA: Contrast meets speed

W.A. Willinek; Bonn/DE

Contrast-enhanced MR angiography (CE-MRA) has been established as an accurate non-invasive method for the diagnostic assessment of the vascular system. However, many clinical applications are still constrained by basic limits on imaging speed. Parallel MR imaging and methods of view sharing in $\mathrm{k}$-space helped to overcome these constraints by enabling 4D time-resolved MRA at very high frame rates. Small, compact and sharper bolus profiles of the intravenously injected contrast agent are favourable for time-resolved MRA in order to obtain optimal contrast-tonoise ratios. Highly concentrated Gadolinium-chelates ( $1.0 \mathrm{~mol} / \mathrm{l}$; Gadobutrol) were described to provide optimised bolus geometries for accelerated MR angiography including highest T1-shortening for small volumes.
Learning Objectives:

1. To learn how to take advantage of highly concentrated contrast-agents for dynamic MRA in clinical applications.

2. To compare the results with those obtained with standard $0.5 \mathrm{M}$ agents.

3. To describe the merits and limitations of new technical developments for 4D time-resolved contrast-enhanced MRA.

\section{D-05}

Closing remarks

V. Runge; Temple, TX/US

$12: 30-13: 30$ Room P

organised by SuperSonic Imagine

SY 3

MultiWave $^{\mathrm{TM}}$ technology introducing ShearWave ${ }^{\mathrm{TM}}$ elastography

Programme not available by date of print

$14: 00-15: 30$ Room C

jointly organised by Siemens and Bayer Schering Pharma

SY 4

Synergies in CT - for better patient care

Moderators:

E.K. Fishman; Baltimore, MD/US

D. Fleischmann; Stanford, CA/US

Y. Imai; Isehara/JP

Z. Jin; Beijing/CN

M.F. Reiser; Munich/DE

U.J. Schoepf; Charleston, SC/US

D-06

Welcome

\section{$D-07$}

High pitch scanning in challenging patients

H. Alkadhi; Zurich/CH

Various strategies exist to reduce the radiation exposure of CT. Most of these, such as prospectively ECG-gated CT, were developed for low-dose cardiac imaging. The most recent one, high-pitch mode, was introduced with the advent of secondgeneration dual-source 128-slice CT. With this, data is acquired in a spiral-mode while the table runs with a high pitch of up to 3.4. This mode can be used - when synchronized with the ECG - for cardiac imaging, enabling a high accuracy at dose of $\sim 1 \mathrm{mSv}$. Another potential application of this mode is lung imaging. CT is the principal diagnostic technique for detailed imaging of lung parenchyma. With early scanners, chest CT was associated with deteriorations in image quality due to non-continuous volume coverage caused by inconsistent levels of inspiration and motion. Thereafter, implementation of spiral scanning allowed for imaging the entire chest in a single breath-hold with scan times between $6 \mathrm{~s}$ and $25 \mathrm{~s}$, thus reducing artifacts. Using the high-pitch mode, the scan-time for the entire chest can be reduced to $\sim 1 \mathrm{~s}$. This mode has the potential to obviate the need for breath-holding, which is relevant in critically ill or non-compliant patients. In deed, high-pitch CT of the lung has shown to provide a diagnostic visualization of the lung even during continuous breathing. Our results indicate that image quality of this mode during breathing was even superior to that of a standard spiral chest CT acquired during deep inspiration breath-hold. Finally, the mode is associated with a very low radiation dose of on $\sim 1.5 \mathrm{mSv}$.

Learning Objectives.

1. To understand the technical background of high-pitch scanning with dual-source 128-slice CT.

2. To learn about the potential of high-pitch scanning for the critically ill and/or non-compliant patient.

3. To appreciate the tremendous radiation dose savings of the high-pitch mode. 


\section{D-08}

Sub-mSv cardiac imaging in clinical routine

J. Hausleiter; Munich/DE

Computed tomography has become a frequently used diagnostic modality for evaluation of the coronary arteries. Nonetheless, there remains concern regarding the associated exposure to ionizing radiation. Besides several established techniques and algorithms for radiation dose reduction, a new high-pitch scan mode has been introduced with the $2^{\text {nd }}$ generation dual-source CT systems. This scan technique allows for a table speed during examination of $43 \mathrm{~cm} / \mathrm{s}$ and thus a typical cardiac scan range of $120 \mathrm{~mm}$ can be examined in $<300 \mathrm{~ms}$ (one single diastolic phase of the cardiac cycle). Initial studies demonstrate that this high-pitch mode allows for a very high level of diagnostic image quality in $99.6 \%$ of all coronary artery segments. Mean DLP was $114 \pm 46 \mathrm{mGy}^{*} \mathrm{~cm}$. In summary, this scan algorithm is a very promising method for low-dose CCTA. Iterative image reconstruction algorithms are currently developed as a novel image reconstruction algorithm for computed tomography which may serve as an alternative to traditional filtered backprojection image reconstructions. As iterative image reconstruction algorithms may decrease image noise, equivalent image quality may be achievable with reduced absolute photon number, thereby permitting reduction in overall radiation dose. The combination of high-pitch CCTA with new iterative image reconstruction algorithms may allows for "sub-millisievert" cardiac CT examinations in a large proportion of patients. The consequent and appropriate use of dose-saving strategies for cardiac $\mathrm{CT}$ is required for best diagnostic studies with lowest possible radiation exposure in every patient. New CCTA image acquisition and reconstruction algorithms may allow for "sub-millisievert" cardiac CT angiographies.

\section{$D-09$}

\section{CT going beyond morphology}

\section{H.-C. Becker; Munich/DE}

Dual Energy CT is a new technique that enables selective imaging and quantification of different elements such as iodine, uric acid and iron. lodine visualization, for instance, is been utilized to display blood volume distribution in different organs, and has found to be of particular additional clinical value in the lung for the detection of pulmonary embolisms and perfusion deficits. Furthermore, dual energy CT with Xenon, used as an inhaled contrast agent, may provide information about lung ventilation, similar to scintigraphy. The malignancy of lung lesions and incidental adrenal masses may be determined by dual energy quantification of the iodine uptake. Furthermore, quantitative dual energy assessment of the myocardial enhancement may also provide information about potential ischemia. In other clinical circumstances, such as in stroke, CT perfusion is already clinically well established. In particular whole brain perfusion with absolute quantitative parameter maps, provides information, that is already been used for immediate triaging. CT perfusion of transplanted kidneys has been proven to provide information about the vascularity as well as the function abnormalities, indicative for rejection, with minimal doses of contrast. For critical cases of lower limb ischemia, time resolved CT angiography provides information about the run off and patency of smallest peripheral arteries. Time resolved CT angiography also provides information about the vascularization and blood supply of the aorta and their branches in case of dissection and endoleakage. All these new techniques may be used an integral part of routine investigations and have the potential to specify the diagnostic accuracy of solely morphologic assessment by CT.

\section{$D-10$}

A guide towards optimising contrast administration in Flash CTangiography

\section{A.H. Mahnken; Aachen/DE}

Only a decade ago multislice CT revolutionized clinical practice. CT-angiography became a cornerstone of CT imaging and new indications like aorto-peripheral and coronary CT-angiography were established in clinical routine practice at breath-taking speed. At the same time the average patients is getting older and more morbid. Increasing complexity of examinations and patients resulted in the demand for optimized contrast regimen aiming on uncompromised image quality achieved with a minimum of contrast material exposure. Most recent developments in CT technology provide the technological basis for minimizing contrast material exposure while maintaining excellent image quality with high contrast-to-noise ratio. The combination of low tube voltage examination protocols with iterative reconstruction technology is an appealing concept for enhancing contrast and reducing image noise, thereby minimizing the amount of contrast material needed. The greatest potential, however, lies within a further reduction of scan time. With recent dual source CT technology high pitch image acquisition became feasible for whole body and cardiac CT. Subsecond scan times became routine practice. Applying the concept of the iodine delivery rate permits the easy translation of the reduced scan times in optimized contrast injection protocols. With these techniques CT-angiography requires only a fraction of the contrast volume that was required only decade ago. Contrast exposure as one of the major contraindications for CT-angiography in an elderly and morbid population will be overcome by the consequent use of these new techniques. Considering these exciting developments more and more patients become eligible for CT angiography, while image quality is further improved. Learning Objectives:

1. To understand the effect of new scanner technology on contrast enhancement. 2. To learn concept of iodine delivery rate.

3. To learn how to design contrast injection protocols for high end CT-angiography

\section{$D-11$}

Best image quality acquired at lowest dose

E.K. Fishman', D. Fleischmann², Y. Imai ${ }^{3}$, Z. Jin ${ }^{4}$, M.F. Reiser ${ }^{5}$, U.J. Schoepf 6 ; ${ }^{1}$ Baltimore, MD/US, ${ }^{2}$ Stanford, CA/US, ${ }^{3} /$ sehara/JP, ${ }^{4}$ Beijing/CN, ${ }^{5}$ Munich/DE, ${ }^{6}$ Charleston, SC/US 


\section{Saturday, March 6}

jointly organised by Bracco and GE Healthcare

\section{SY 5}

\section{Technological development and current role of contrast enhanced ultrasound}

Moderator:

J.-M. Correas; Paris/FR

\section{$D-12$}

Introduction

J.-M. Correas; Paris/FR

\section{$D-13$}

Technological evolution and current role of CEUS in clinical imaging

E. Leen; London/UK

\section{$D-14$}

CEUS in focal liver lesions examination

V. Vilgrain; Clichy/FR

\section{$D-15$}

CEUS and high frequency imaging

E.M. Jung; Regensburg/DE

\section{$D-16$}

Conclusion

J.-M. Correas; Paris/FR

$$
12: 30-13: 30
$$

\section{organised by Guerbet}

\section{SY 6}

\section{CT contrast media optimisation for patient benefit \\ Moderator:}

M.H. Maurer; Berlin/DE

\section{$D-17$}

Introduction

M.H. Maurer; Berlin/DE

\section{$D-18$}

Main parameters involved in CIN: Is viscosity the main one?

L. Juillard; Lyon/FR

Purpose: Properly controlled clinical studies of intravenously administered radiographic contrast media fail to demonstrate renal damage. No scientifically rigorous data exist to support the nephrotoxicity of contrast material. In addition, there is stil a matter of debate whether the use of iso-osmolar contrast media as opposed to the use of low-osmolar contrast media would be associated with a lower incidence of contrast media-induced nephropathy $(\mathrm{CIN})$.

Methods and Materials: We performed a MedLine literature search (1966 to 2004) to identify studies assessing the nephrotoxicity of contrast material. A total of 3081 publications were found. Among them 40 (1.3\%) were performed with intravenous injection and 2 had a group control with no injection. CIN incidence was defined as a serum creatinine ( $\mathrm{SCr}$ ) decrease of $25 \%$ or more (or $\geq 44 \mu \mathrm{mol} / \mathrm{l}$ ) from baseline, within 3 days after injection, and in the absence of other causes (e.g., atheromatous embolic disease, ischemia, other nephrotoxins).
Results: These studies showed that contrast agents by intravenous (iv) may be a lower risk, CIN incidence was reported in 3 to $9 \%$ of patients, there was a very low risk for dialysis, and few studies of renally impaired patients getting low- or iso-osmolal media IV. Several risk factors for CIN have been identified mainly renal insufficiency, age, diabetes, hypotension, low cardiac output.

Conclusion: The main prevention approaches were the use of low- or iso-osmolar contrast media, adequate hydration (IV or oral, saline or bicarbonate, dose and duration), anti-oxidant (theophylline, ascorbic acid, N-acetylcysteine), hemofiltration, vasoactive agents (dopamine, fenoldopam), avoid diuretics (furosemide, mannitol), and stop administration of nephrotoxic drugs for at least 24 hours. Anyway, this literature search showed that there were many inadequate trials.

\section{$D-19$}

Comparative study of low osmolar contrast media in pediatric renal tolerance

M. Hörmann; Vienna/AT

Purpose: lodinated contrast media-induced nephropathy $(\mathrm{CIN})$ is one of the most common reasons for hospital-acquired acute kidney injury. There is still a matter of debate whether the use of iso-osmolar contrast media as opposed to the use of low-osmolar contrast media would be associated with a lower incidence of CIN. Methods and Materials: 146 children, 11 months to 16 years old, included in this multicenter trial underwent enhanced CT for any indications and received either a mean injection of $1.8 \pm 0.4 \mathrm{ml} / \mathrm{kg}$ Xenetix ${ }^{\circledR} 300$ (iobitridol) or $1.9 \pm 0.2 \mathrm{ml} / \mathrm{kg}$ Visipaque $₫ 270$ (iodixanol). The primary endpoint was the relative variation of the creatinine clearance between pre (48h) and post-injection (72h) of contrast product, using a non-inferiority analysis. Secondary endpoints were the incidence of CIN (defined as a creatinine clearance decrease of $25 \%$ or more), global contrast quality (3-point scale: poor, moderate, good), diagnostic efficacy (3-point scale: impossible, difficult, easy) and clinical safety (adverse events and vital signs).

Results: Non-inferiority for the intent-to-treat population (128 children) was demon strated when considering the $90 \%$ two-sided confidence interval $[-0.0481 ; 0.0456]$ the lower confidence limit was superior to the non-inferiority margin $(-0.05)$. The results were consistent in the per-protocol population ( 68 children). CIN incidence was observed in 3 patients (4.8\%) with iobitridol and 7 patients $(10.6 \%)$ with iodixanol. No statistical significant differences were observed for the other secondary criteria. Conclusion: Similar and good safety profile of both products was confirmed, with out significant effect on the CIN occurrence in children with normal renal function.

\section{$D-20$}

Xenetix®: Excellent tolerance confirmed by 160.000 additional patients M.H. Maurer; Berlin/DE

Purpose: The purpose of this study was to review the safety, the tolerability and the diagnostic effectiveness of iobitridol under daily practice conditions using different IV application types. The study of the general population and, in particular, of at-risk patients was based on a surveillance study carried out in Germany from 2002 to 2008.

Methods and Materials: A total of 160,639 patients (55.1\% male, $43.6 \%$ female mean age 58.6 years) were analysed in 555 centers. Patients underwent X-ray examinations using iobitridol (Xenetix ${ }^{\circledR}$, Guerbet) as IV contrast medium. A new polypropylene infusion bag (ScanBag ${ }^{\circledR}$ ) was thereby deployed in 17,614 patients. $21.8 \%$ of all patients had at least one risk factor (e.g., renal impairment, hypo- or hypertension, diabetes mellitus, coronary heart disease), $7.3 \%$ were at-risk patients with allergies or who had previously reacted to contrast medium. 1,114 patients $(0.7 \%)$ were given a preliminary antiallergic medication prior to the study. lodine concentration was either $300 \mathrm{mg} / \mathrm{ml}(144,316$ patients) or $350 \mathrm{mg} / \mathrm{ml}(15,328$ patients) with a mean volume of $85.6 \mathrm{ml}$. In addition, height and weight for each patient were documented and the image quality was assessed.

Results: A diagnosis was possible in $99.5 \%$ of all cases. The image quality was assessed good or excellent in $92.2 \%$. The main reason for a poor image quality $(0.2 \%)$ was obesity. In obese patients, the use of iobitridol 350 improved the percentage of examinations with good or excellent quality from $92.8 \%$ to $97.2 \%$. The adverse event rate (e.g., nausea, urticaria, pruritus) observed was $0.6 \%$ in all patients, $1.6 \%$ in patients with allergies and $6.0 \%$ in patients with a previous reaction to contrast medium. Pretreatment did not decrease the rate of adverse events. Furthermore, the rate of adverse events was not increased in the highe dose of lobitridol $(350 \mathrm{mg} / \mathrm{ml})$, even if this was administered to high risk patients. Conclusion: lobitridol was shown to be a well-tolerated contrast medium resulting in good or excellent image quality in most patients also using polypropylene bags as a new IV application type. The incidence of adverse events was low and not related to prior administration of pretreatment. 


\section{$D-21$}

Optimised contrast media management in cardiac CT

J.-F. Paul; Le Plessis-Robinson/FR

Purpose: Optimisation of contrast media injection for cardiac and vascular examinations.

Methods and Materials: Injection time should be adapted to the acquisition time to avoid excess of contrast delivery. Acquisition time is becoming shorter and shorter with evolving technology of multislice CT: 5 seconds using 64-slice CT for a whole thorax and $<1$ second using the lastest generation of CT for a whole thoracic aorta. Because of this short temporal window, monitoring the transit time using test bolus or automatic detection techniques is now mandatory to customize contrast delivery. Sufficient enhancement is also very important to ensure cardiac and vascular structures visualisation. Small vascular structures like coronary arteries require higher vascular contrast for clear delineation. However with too high contrast, vascular calcifications may be hidden by contrast, if densities are similar Results: Ideally, a target value should be defined by the user for each clinical situation. Two main categories of parameters are to be considered for reaching this target: Not patient-related: - Flux of iodine per second (resulting from iodine concentration and injection rate). - Kilovoltage: lower kilovoltage is responsible for higher enhancement, due to increased photo electric effect. Patient related: - Weight, body size and cardiac output are crucial parameters influencing vascular enhancement. Conclusion: Knowledge of these parameters makes possible to optimize contrast delivery on an individual basis, and this approach is under investigation with iobitrido 350 on patients undergoing enhanced cardiac CT. Also, computerized programs may be helpful for reaching targeted contrast enhancement in most cases.

\section{$D-22$}

Questions and conclusion

M.H. Maurer; Berlin/DE

$$
\text { 12:30 - 13:45 }
$$

Room C

\section{organised by GE Healthcare}

\section{SY 7}

\section{New and efficient case-solving tools in daily breast and chest procedures}

Moderator:

L. Katz; Buc/FR

\section{$D-23$}

Clinical experience with dual-energy contrast-enhanced digital mammography (CEDM) for detection of breast carcinomas C. Dromain ${ }^{1}$, F. Thibault², C. Malhaire², M.C. Mathieu', S. Delaloge', C.S. Balleyguier'; ${ }^{2}$ Villejuif/FR, ${ }^{2}$ Paris/FR

Purpose: To present the dual energy CEDM technique, preliminary clinical performance and potential clinical applications.

Methods and Materials: CEDM is a new application of digital mammography using intra-venous injection of an iodinated contrast agent in conjunction with a mammography examination (MX). 107 women with 133 suspicious findings on MX and/ or ultrasonography underwent CEDM. A pair of low and high energy images was acquired using a modified full-field digital mammography system. Exposures were taken in MLO view at 2 minutes and in CC view at 4 minutes after the injection of $1.5 \mathrm{ml} / \mathrm{kg}$ of an iodinated contrast agent. One experienced breast radiologist interpreted $\mathrm{MX}$, then $\mathrm{MX}+\mathrm{CEDM}$ images for all cases, blinded on clinical information. The gold standard was the pathology results in all cases.

Results: Pathology identified 80 benign and 53 malignant lesions. The sensitivity was $0.94,0.94,0.94$ for $M X+C E D M$ compared to $0.80,0.78,0.85$ for $M X$ alone considering, respectively, probability of cancer $(p=0.01)$, BI-RADS $(p<0.01)$ and confidence of presence $(p=0.07)$. There were no significant differences between specificity for $M X$ and $M X+C E D M$. The area under the ROC curve was superior for $M X+C E D M(p<0.01)$, using either probability of malignancy or BIRADS. The potential clinical applications are the determination of the extent of disease, the assessment of recurrent disease, the clarification of mammographically equivocal lesions, the detection of occult lesions on standard mammography, particularly in dense breast, and the monitoring of the response to chemotherapy.

Conclusion: CEDM should be a useful adjunct to diagnostic mammography and a promising problem-solving and staging tool.

\section{$D-24$}

The value of digital tomosynthesis in the diagnosis of thoracic lesions E. Quaia; Trieste/IT

The detection and characterization of pulmonary lesions, and particularly of pulmonary nodules, are challenging tasks in thoracic imaging due to their frequent small size and poor conspicuity within surrounding anatomical structures. Digital tomosynthesis is a tomographic technique like $\mathrm{CT}$, but it delivers a lower radiation dose and is easily implemented in conjunction with chest radiography as it employs the same X-ray equipment. Digital tomosynthesis is decisive for differentiating thoracic opacities due to parenchymal lesions from those due to the pleural or thoracic wall lesions or pseudolesions. In our department, digital tomosynthesis was easily introduced in the routine diagnostic work-flow and applied only in most patients with suspected or equivocal lung lesion of frequent identification at chest radiography. The use of tomosynthesis entails less waiting time and radiation dose than sending the patients directly to CT for the workup of suspected lung parenchyma lesions that turn out to be benign, nonpulmonary, or nodule mimics. Once a chest opacity is identified as being a pulmonary lesion by tomosynthesis, it generally goes to CT for further evaluation, classification, and staging. Tomosynthesis obviates the need for the lateral projection, in which case the total exposure including tomosynthesis is slightly superior to a conventional examination performed with posteroanterior plus lateral view. A further field of application of tomosynthesis could be the follow-up of pulmonary nodules previously identified on chest radiography or CT. This approach would save radiation dose and reduce costs compared with following-up the nodules with periodic CT scans.

\section{$D-25$}

Digital chest tomosynthesis in daily clinical practice

M. Boijsen, Å.A. Johnsson, A. Svalkvist, S. Zachrisson, A. Flinck, S. Kheddache, L.G. Månsson, M. Båth, J. Vikgren; Gothenburg/SE

Since December 2006, more than 3000 chest tomosynthesis examinations (TS) (VolumeRad, GE), including two pulmonary nodule detection studies, enrolling 114 consenting patients, have been performed at our institution at Sahlgrenska University Hospital, Göteborg, Sweden. The most frequent indications for TS have been suspicion of a nodule, tumour, pneumothorax, cavitation or possible confounding skeletal pathology seen on conventional chest radiography (CR). CR suffers from low sensitivity and specificity. Patients are often referred to computed tomography (CT) for further work-up of suspicious findings. CT often solves the problem, but has disadvantages such as high radiation dose and high costs. TS offers an improved chest radiography method, which can be used to select patients who need further investigation with $\mathrm{CT}$, thereby reducing the radiation dose to the patient population and optimising the use of available CT resources. However, TS showed some limitations, such as the requirement for patients to hold their breath for $10 \mathrm{~s}$ and limited depth resolution of reconstructed images. In the clinical setting at our institution, TS is now often used as an additional examination to $\mathrm{CR}$ and to decide whether to recommend CT or not based on the findings of the combination of CR and TS. TS is also used for follow-up of pathology that has been confirmed by $C T$ in selected cases and is also sometimes requested by the referring clinicians as the "method of choice" for chest examinations.

12:30 - 13:30

Room F2

organised by Hitachi

SY 8

Programme not available by date of print

$12: 30-13: 30$ Room P

organised by Philips Healthcare

SY 9

Programme not available by date of print

$12: 30-13: 30$ Room Q

organised by Philips Healthcare

SY 10

Programme not available by date of print 


\section{organised by Hologic}

\section{SY 11}

\section{Breast tomosynthesis: Clinician experiences with the first commercial system \\ Moderator:}

A. Smith; Bedford, MA/US

\section{$D-26$}

A comparison of the accuracy of digital breast tomosynthesis, film-screen mammography, and digital mammography

M.J. Michell; London/UK

\section{$D-27$}

Evaluation of digital breast tomosynthesis for use in a national screening programme

P. Skaane; Oslo/NO

\section{$D-28$}

Vacuum assisted biopsy on a digital breast tomosynthesis system: Initial clinical experience

P. Gignier; Antony/FR

\section{organised by Bayer Schering Pharma}

\section{SY 12}

\section{From molecular imaging to histopathology MRI}

Moderator:

J. Ricke; Magdeburg/DE

\section{$D-29$}

Introduction

J. Ricke; Magdeburg/DE

\section{$D-30$}

Imaging of dementia today and tomorrow

G. Frisoni; Brescia/IT

Degenerative dementias have been recognized as being due to abnormal accumulation of toxic proteins such as beta amyloid, hyperphosphorilated tau, and alpha synuclein. This concept opens new avenues for diagnostic imaging. The case of Alzheimer's is prototypical in this respect. Traditional NINCDS[[Unsupported Character - \&\#8208;]]ADRDA criteria allow to diagnose Alzheimer's disease (AD) at the progressed dementia stage with a very limited amount of instrumental data and are believed to have a clinically satisfactory sensitivity and specificity. Recognizing $A D$ at earlier stages is a much more uncertain exercise. Data accumulated over the past 10 years suggest that patients with mild cognitive impairment and structural or metabolic markers consistent with AD have a high chance of disease being present and of developing dementia within the following 5 years. Structural markers comprise atrophy of medial temporal structures such as the hippocampus, amygdala, and entorhinal cortex on MRI, and metabolic markers temporoparietal hypometabolism on glucose PET. The lack of standard operational procedures to measure markers and their unknown combination is a significant hurdle for their clinical implementation. Recently, tracers for cerebral amyloid have been developed (PIB, FDDNP, florbetaben) allowing to directly appreciate amyloid deposition, which is believed one of the major pathogenetic factors. While amyloid imaging might be a sensitive marker of $A D$ in patients with mild cognitive impairment, the high prevalence of positivity in apparently healthy elderly raises questions about diagnostic anticipation.

Learning Objective:

1. To learn about current and future imaging techniques for the early and differential diagnosis of the neurodegenerative dementias.

\section{$D-31$}

Liver cell-specific imaging reaching out to histopathology J. Ricke; Magdeburg/DE

Successful treatment of hepatocellular carcinoma (HCC) is complicated by the fact that early-stage disease is mostly asymptomatic, and many tumors are identified at an advanced stage. The aim of diagnostic imaging is to detect HCC earlier, when curative options are available, and thereby improving patient management and prognosis. The sensitivity of MRI can be increased through the use of contrast agents. New MR contrast agents have been developed that are highly specific for liver cells. These improve diagnostic sensitivity through exclusive uptake by hepatocytes, but not tumors lacking normal liver cell function. Currently available hepatocyte-specific contrast agents vary in their performance profile with respect to signal intensity and the time required for optimal enhancement. Agents with fast and efficient uptake by hepatocytes improve diagnostic confidence for the radiologist as well as convenience for the patient. In HCC diagnosis, specifically differentiation of $\mathrm{HCC}$ from premalignant lesions such as dysplastic nodules is challenging. Typically malignant lesions can be identified through increased enhancement (hyperintensity) during the arterial phase (wash-in) and decreased enhancement (hypointensity) during the portal venous phase (wash-out), when the surrounding liver parenchyma is enhanced. In addition to these criteria, late phase imaging such as lesion uptake of the hepatocyte-specific contras agent, or the character in T2w sequences, greatly improve not only the depiction of malignant nodules, but also the diagnostic confidence for early detection and secure characterization of premalignant lesions. Clinical case examples will be presented, demonstrating how hepatocyte-specific MRI influences treatment decisions to improve overall HCC patient management.

Learning Objectives:

1. To understand the properties of hepatocyte-selective contrast agents.

2. To enable the clinical differentiation between malignant and premalignant lesions in cirrhotic livers.

\section{$D-32$}

The added value of Primovist (Gd-EOB-DTPA) in the diagnosis and treatment of HCC

A. Filippone; Chieti/IT

Hepatobiliary contrast agents are taken up to varying degrees by functioning hepatocytes and are excreted in the bile. Hepatocytes in well-differentiated hepatocellular carcinoma may retain enough hepatocellular function to take up hepatobiliary contrast agents, may appear iso- or hyperintense to liver at delayed imaging, and hence may be overlooked at this phase of imaging. Gd-EOB-DTPA is a new hepatobiliary contrast agent, recently introduced for clinical imaging. After a bolus injection, Gd-EOB-DTPA behaves as a non-specific extracellular contrast agent, and, therefore, it can be used also for dynamic contrast-enhanced MR sequences. Currently, dynamic contrast-enhanced imaging added to hepatobiliary phase remains the current mainstay for HCC detection: some investigators have suggested that Gd-EOB-DTPA is superior to 64-MDCT in detecting $\mathrm{HCC} 1 \mathrm{~cm}$ in diameter or smaller. Therefore, the vascularity and hepatocellular-specific properties of a lesion can be concurrently evaluated in one examination, and this allows to better characterize any lesions in cirrhotic liver. Moreover, the hepatobiliary phase, alone, offers the possibility of defining the differentiation grade of $\mathrm{HCC}$ nodules, which has an important clinical impact. Following the detection and characterization of pre-malignant lesions and well-differentiated HCC, a widening of inclusion criteria for radical therapies and an early inclusion of high-risk patients in the waiting list for liver transplantation could improve the long-term post-treatment results. Learning Objectives:

1. To describe the mechanism of action of hepatobiliary-specific contrast agents 2. To review the enhancement patterns of $\mathrm{HCC}$ nodules at the hepatobiliary phase and underline their diagnostic value.

3. To discuss the clinical usefulness of adding the hepatobiliary phase to the dynamic contrast-enhanced imaging in the management of HCC nodules.

\section{$D-33$}

Dual excretion pathway paving the way for biliary imaging E.M. Merkle; Durham, NC/US

The goal of this lecture is: 1 . To familiarize the radiologist that hepatobiliary contrast agents such as Gd-EOB-DTPA can be used in the dynamic functional evaluation of the hepatobiliary system. 2. To familiarize the radiologist that excretion of Gd-EOBDTPA into the biliary system can be seen within 20 minutes post-injection in patients with normal bilirubin levels. 3. To familiarize the radiologist that Gd-EOB-DTPA can be used to differentiate post-surgical fluid collections from bilomas, as the identification of a hepatobiliary contrast agent within a fluid collection on delayed images can indicate communication with the biliary tree. 4 . To familiarize the radiologist that biliary excretion of Gd-EOB-DTPA helps to evaluate the cystic duct patency. 


\section{organised by Siemens}

\section{SY 13}

\section{Open new ways of imaging in radiology \\ Moderator:}

M. Forsting; Essen/DE

\section{D-34}

\section{Redefining productivity in MR}

M. Forsting; Essen/DE

Statement of problem: The increasing world-wide proliferation of MRI systems has made this valuable diagnostic technology abundantly accessible. However, this rapid growth has followed an even higher increase in demand and the requirement to accommodate a larger variety of patient conditions and complex indications. Furthermore, the availability of skilled MR operators has not met the need within the industry. Finally, the variety and increased complexity of techniques and imaging sequence options has, in some cases, led to large variations in imaging results. Objectives of programme: 1.) To demonstrate how the implementation of advanced technology can improve productivity in an MRI practice; 2.) To demonstrate how the implementation of advanced technology can improve image quality and consistency across a large variety of patients and MRI operator experience; 3.) To demonstrate how the standardization of protocol derivatives (e.g. changing protocols due to patient needs) can improve overall image quality in an MRI practice with a large variety of patient indications and conditions; 4 .) To provide the learner with examples of clinical cases where productivity and image quality were improved by the incorporation of advanced technology.

Description of programme: I plan to demonstrate various examples of new hardware and software technology in coordination with clinical cases and how these advances can be incorporated to benefit all stake holders (e.g. patient, referring physician, technologist, radiologist, etc.) in a clinical environment.

\section{$D-35$}

\section{MR from head to toe with TimCT}

M. Lentschig; Bremen/DE

The introduction of Tim (Total imaging matrix) revolutionized the concept of mult station imaging techniques some years ago. But, one of the latest technologica advancements in magnetic resonance imaging by Siemens is syngo TimCT, which offers continuous table move for scanning, like computed tomography. This allows physicians to acquire a complete scan within a single, continuous move. With syngo TimCT, the table is not required to stay in a fixed position to acquire images. The table moves continuously as it does during a CT scan, while TIM enables the signa to be acquired simultaneously. This results in faster scans, increased image quality and the potential for new application capabilities. Today, syngo TimCT is available as TimCT Onco for oncologic questions and as TimCT Angio as a new MR angiography technique. The advantages of this new scanning technique are scanning up to whole body imaging, seamless acquisition, large total FoV but small active FoV. In combination with "AutoCoilSelect", TimCT improved patient throughput and technicians' workflow. The purpose of this talk is to give an overview from clinicians' perspective how we can use the TimCT technology in clinical practice and what the benefits for the patient are. With TimCT, whole body imaging, especially for cancer patients, will become a practical reality because of rapid acquisition including Dixon techniques, post contrast measurements and fusion of PET information with magnetic resonance imaging. It has been shown that a reduced scanner length combined with Open Bore can improve patient comfort and acceptance.

\section{$D-36$}

Molecular CT: Radiological aspects in hybrid imaging

G. Antoch; Essen/DE

Hybrid imaging systems have become widely available. These scanners provide morphological and functional data in a single session and have been found to be more accurate for tumor staging than morphology or function alone for a variety of malignant diseases. While the collection and interpretation of CT image sets represent a core competence of radiologists, PET and SPECT imaging clearly fall within the realm of nuclear medicine. Thus, combining the two methods requires close co-operation between radiology and nuclear medicine to assure the maximum diagnostic yield by optimizing both the $\mathrm{CT}$ and PET components. The most recently launched line of PET/CT scanners combines high-definition PET with high-end

multislice CT. These imaging systems not only provide a higher diagnostic accuracy based on detection of smaller lesions with CT and PET, they also offer integration of complex CT protocols into the PET/CT scan. These protocols include CT perfusion, three-dimensional CT image reconstruction, virtual fly-through, or "anatometabolic" cardiac data sets. Radiological expertise is an essential precondition to optimize CT imaging protocols in PET/CT and to assess these "molecular CT" data sets. The aim of this talk is to give an overview summarizing new developments in PET/ $\mathrm{CT}$ technology and to address radiological aspects regarding PET/CT imaging. Learning Objectives:

1. To summarise new developments in PET/CT imaging.

2. To define the role and responsibility of the radiologist in hybrid imaging.

3. To understand the benefit of optimising the morphological part in hybrid imaging

\section{$D-37$}

Clinical PET/CT beyond FDG

P. Bartenstein; Munich/DE

$\mathrm{PET} / \mathrm{CT}$ has in recent years exhibited the highest growth rate of all clinical imaging modalities. To date, the vast majority of all PET/CT investigations are still performed with [18F]fluorodexoxglucose (FDG). However, more specific radiopharmaceuticals are being developed at a rapid pace. At the LMU of Munich, the percentage of clinical non-FDG patient studies has increased from $7 \%$ to over $30 \%$ in four years. Detection of proliferating cells within tumours can be obtained in PET/CT studies with the thymidine analogue [18F]-FTL, which provides accurate information about therapeutic response. The delineation of hypoxic domains within tumours is revealed with agents such as [18F]-misonidazol, which can improve radiation therapy planning. PET/CT with the amino acid [18F]-fluoroethyltyrosine, a surrogate marker of protein synthesis, has emerged as the diagnostic standard for the detection of recurrent brain tumours and biopsy planning. [18F]-labelled choline derivatives have gained clinical importance for the initial and re-staging of prostate cancer. The judicious combination of diagnostic and therapeutic radiopharmaceuticals constitutes a further advancement. In particular, the expression of somatostatin receptors in some neuroendocrine tumours can be measured with the receptor antagonist [68Ga]-DOTATATE. In receptor positive cases non-trace doses of beta-emitting somatostatin antagonists can be used for targeted therapy. It is likely the next years shall see the emergence of new clinically important radiopharmaceuticals for monitoring other relevant tumour properties, such as angiogenesis or apoptosis. Learning Objective:

1. To understand use of non FDG-PET.

$14: 00-15: 30$ Room N/O

organised by Sectra

SY 14

How do we improve cancer detection rates in mammography today?

Moderators:

M. Danielsson; Stockholm/SE

H. Ringertz; Linköping/SE

\section{$D-38$}

Why do we miss cancers in mammography: What the DMIST trial tells us M.J. Yaffe; Toronto, ON/CA

Background: In the Digital Mammographic Imaging Screening Trial, digital mammography performed better in diagnosing cancer in women with dense breasts, women younger than age 50, and pre- and perimenopausal women, whereas screen-film mammography performed better in women older than age 65 and in women with fatty breasts. We studied which imaging factors contributed to the better accuracy of digital mammography.

Methods: Seven radiologists compared the digital with the screen-film mammogram for each lesion. All knew each mammogram showed malignancies, al had acetate overlays showing the location of the lesion, and Breast Imaging and Reporting System descriptors for each lesion. Each radiologist scored the visibility of each lesion on a 5-point visual analog scale, and the scores were sorted into 1 of 4 categories per case indicating whether the lesion was more visible on the digital or the screen-film mammogram, equally visible, or not visible on either type. Results: Opinions and reasons for the differences in visibility varied considerably. However, for cancers in dense and in fatty breasts, the most frequent reason was contrast differences. For dense breasts, contrast differences accounted for almost $20 \%$ of the 378 reasons; other reasons ranged from $0 \%$ to $9 \%$. Position, compres- 
sion, and differences in technique accounted for only $10 \%$ of the reasons. Conclusions: The better diagnostic accuracy of digital mammography was most likely related to differences in image contrast, which in turn were most likely produced by the acquisition and display characteristics of the technology, rather than to differences in positioning, compression, or reader skill.

\section{D-39}

Tomosynthesis imaging of the breast: Challenges and opportunities M.G. Wallis; Cambridge/UK

Breast tomosynthesis is proposed as a possible new method in mammography to increase sensitivity and specificity. By scanning the breast and acquiring images in a number of projections in different angles it is possible to reconstruct a 3D image constituted by slices of arbitrary thickness that can be viewed individually. This may help detection of cancers which would otherwise have been shadowed by overlaying tissue and it may also reduce call backs in cases where overlapping tissue mimic malignant tissue. A review of the currently available and proposed techniques for tomosynthesis breast imaging will be given together with detector technology and scanning geometries In particular cases from the European Union supported Highrex project will be shown as examples of opportunities and pit-falls with the new technique. Also possible implementations in a screening environment will be discussed including the possible future need for traditional 2D images.

\section{$D-40$}

\section{Contrast mammography: How is it done and how can it help}

F. Diekmann; Berlin/DE

Breast MRI has matured to a common imaging procedure in many countries. Normally Gadolinium is injected as contrast agent to visualize the enhancement of tumors. Blood vessels related to the cancer are claimed to be of low quality and leaky which allows tumor visualization. Also take-up and wash-out curves of the contrast agent in suspicious lesions can be measured. There is today significant evidence that breast MRI adds important information in diagnostic follow up and it is also proposed for screening of high risk groups. lodine is the gold standard for contrast agents in x-ray imaging today and is for example used in more than half of all CT examinations. The K-edge absorption peak for lodine can be used for imaging with energies from mammography machines, and lately so called contrast mammography has generated significant interest with promising clinical results in several pilot trials. In one technique for contrast mammography consecutive images of the breast are acquired and the lodine signal is singled out through subtraction of a pre-contrast image. The other technique for contrast mammography is based spectral $\mathrm{x}$-ray imaging in which the energy of the $\mathrm{x}$-rays is measured and sorted below and above the absorption edge of the lodine. Also with this method consecutive images may be acquired but there is no need for a pre-contrast reference image. One single image is enough to visualize the lodine, which makes it less sensitive to motion blurring and reduces the dose. Both methods for contrast mammography are expected to reveal the blood vessels of the cancer analogous to breast MRI. Differences and similarities between the methods will be discussed such as image acquisition time, image quality and cost.

\section{$D-41$}

\section{Photon counting mammography in daily practice}

J.-C. Piguet; Geneva/CH

Mammography remains the major tool for screening breast disease, despite the limited sensitivity of the method (75\%). However, this Sensitivity has been improved with introduction of Digital Mammography (DR), especially in dense Breasts. Screening concerns mostly healthy women, in other words this means that $>$ of $99 \%$ of tested women have performed the mammogram for "nothing". It is thus the responsibility of the radiologist to insure to the healthy population as low X-ray exposures as possible. The challenge is to reduce the dose significantly and stil produce excellent image quality and we will discuss this important trade-off and relevant cases will be presented. If the image quality and cancer detection rate is a least as good as any other DR system the question is: Why you should the dose to healthy women with a factor of 2 to 5 without obtaining more relevant information.

\section{Discussion}

14:00 - 15:30 Room Q

organised by Siemens

SY 15

Biopsies in breast imaging: Methods and needs

Moderator:

J. Dick; Erlangen/DE

\section{D-42}

Introduction

J. Dick; Erlangen/DE

\section{$D-43$}

Innovations and challenges in mammography guided biopsies

K. Ridder; Dortmund/DE

In the last years, minimal invasive breast-biopsies have become one of the main tools of diagnostic work. Besides ultrasound as the most common method, stereotactic (Mammographic) and MRI-guided biopsies are done routinely in most breast center sites. Especially for stereotactic guided biopsies, it is important to have a straightforward and successful approach to reduce biopsy-time, discomfort and complications for the patient. From a technical point, automated tube movement and superior picture quality in combination with a bigger detector size are the main goals which have been realized. Prone and upright biopsy systems on the market have their own specificities. But it is more important to preselect the patients and to reduce the number of false-positive results in imaging in order to minimize the number of unnecessary biopsies using modern imaging techniques. Learning Objectives:

1. To establish criteria for appropriate patient selection and management for stereotactic guided interventions.

2. To discuss advantages/disadvantages of different patient-positioning and systems available.

3. How can we avoid unnecessary biopsies by reducing false positive results in imaging?

\section{$D-44$}

Innovations and challenges in ultrasound guided biopsies

F. Stöblen; Essen/DE

Breast imaging and reliable interventional tissue sampling are paramount for the early diagnosis of breast cancer and thus facilitate treatment of the disease in curable stages. In screening settings, mammographically suspicious lesions are usually examined by sonographic imaging and ultrasound-guided biopsy. Technical development in breast ultrasound is mainly targeted at three goals: 1. reliable and economical avoidance of false-positive findings, i.e. unnecessary biopsies, 2 . facilitation of accurate specimen obtainment when necessary, and 3. containment or reduction of physician time without compromise in diagnostic accuracy. Emerging technologies that are currently under experimental and clinical evaluation are automated breast volume scanning and touch elasticity imaging (e.g., ACUSON S2000, Siemens Healthcare). Potential benefits and pitfalls of those methods are presented and future perspectives in ultrasound breast imaging discussed. Learning Objectives:

1. To learn about the use of ultrasound in breast imaging and biopsy.

2. To know advantages of automated breast volume scanning

3. To understand the clinical value of innovative applications such as elastography.

\section{$D-45$}

Breast MRI guided diagnostic interventions

E. Wenkel; Erlangen/DE

The role of breast magnetic resonance imaging (MRI) for diagnosis, screening, staging and management of breast cancer is becoming larger each year. It has proven to constitute a valuable complementary tool to the conventional techniques like physical examination, mammography and ultrasound. Breast MRI is a more sensitive method for the detection of malignant breast lesions than conventional mammography and ultrasound. In literature, the sensitivity of breast MRI for invasive breast cancer has been described as near $100 \%$. However, the specificity of MRI has a wide range from 50 to $95 \%$ especially depending on imaging techniques and patient selection. When suspicious breast findings can only be identified at MRI, MRI guided techniques are used for adequate tissue sampling to exclude breast cancer histologically 


\section{Learning Objectives:}

1. To establish criteria for appropriate patient selection and management for MRI guided interventions.

2. To discuss basic principles and challenges of MRI guided intervention.

3. To understand technical requirements for coil, needle and approach selection

4. To discuss the implication of pathology and follow-up of these patients.

\section{Panel discussion}

\section{Sunday, March 7}

$10: 30-12: 00$

Room P

\section{organised by Bracco}

SY 16

Programme not available by date of print

$12: 30-13: 30$

Room B

organised by Guerbet

SY 17

How to optimise the dynamic use of contrast media in MRI?

Moderator:

C. Ayuso; Barcelona/ES

\section{$D-46$}

Introduction

C. Ayuso; Barcelona/ES

\section{$D-47$}

Main indication in dynamic MR

C. Ayuso; Barcelona/ES

The extracellular, non-specific contrast agents gadolinium chelates are the most widely used in the MRI daily clinical practice. They are relatively safe when used in clinically recommended doses and in this scenario they cause a strong decrease of the $\mathrm{T} 1$ relaxation time and as a consequence, they produce an increase of signal intensity. They add morphologic and functional information compared with unenhanced MRI improving the sensitivity and specificity of MRI studies. The aim of this presentation is to summarize main characteristic, mechanism of action, tolerance profiles and main indications of these agents in normal and high-risk patients, as well as MR technical requirements for dynamic studies enable to enhance the diagnostic effect of these agents.

\section{$D-48$}

3D tricks in intracranial arteriovenous malformations

O. Naggara; Paris/FR

Our aim is to illustrate the value of 1.5 and $3 \mathrm{~T}$ three-dimensional (3D) dynamic time-resolved contrast-enhanced MRA (TR-CE-MRA) using a combination of a parallel imaging technique (ASSET: array spatial sensitivity encoding technique) and a time-resolved method (TRICKS: time-resolved imaging of contrast kinetics) and to compare it with intra arterial digital substraction angiography (DSA) in the assessment of intracranial arteriovenous malformations (AVMs). DSA remains the reference standard for diagnosis, treatment planning, and post therapeutic monitoring of AVMs. However, it is an invasive procedure that involves ionizing radiation and the use of iodinated contrast medium, and is associated with significant morbidity and mortality as patients with AVMs undergo multiple DSAs. 3D dynamic TR-CEMRA combining parallel imaging and a time-resolved method with sub second and submillimeter resolution allow demonstration of abnormal communication between the arterial and venous systems, determine nidus size, the number and type of feeders and drainers with an excellent accuracy. Limitation are in the detection of the presence of vascular aneurysm or venous stenosis. This technique is actually the first-line investigation technique in both diagnosis and follow-up of intracranial AVMs in our institution. 


\section{$D-49$}

Benefit of MRI myocardial perfusion with Dotarem ${ }^{\circledR}$ at 1.5T and 3T P. Bernhardt; Ulm/DE

Purpose: Non-invasive early detection of relevant coronary artery disease in intermediate-probability patients still remains challenging. Different stress tests such as stress-ECG, stress-echocardiography or nuclear imaging are being used, but often lack sufficient accuracy, reproducibility and/or spatial resolution. Cardiovascular magnetic resonance (CMR) combines in one examination information about global and regional left ventricular function, myocardial perfusion and myocardial viability, but little is known about CMR perfusion at higher magnetic field strength Methods and Materials: 41 patients with suspected coronary artery disease were included in this cross-over study. Each patient underwent two consecutive imaging sessions, one at 1.5 T (Intera, Philips Medical Systems) and one at 3T (Achieva, Philips Medical Systems) 48 hours later. Then, standard of reference which was invasive X-ray coronary angiography was performed within 72 hours after CMR. Myocardial perfusion was assessed in rest conditions and after a pharmacological stress using a steady-state free precession (SSFP) or gradient-echo sequence, and perfusion imaging was performed using 40 dynamic acquisitions during the administration of an intravenous bolus of gadolinium chelates $(0.1 \mathrm{mmol} / \mathrm{kg}$ at $1.5 \mathrm{~T}$ or $0.075 \mathrm{mmol} / \mathrm{kg}$ at 3T). During maximum adenosine ( $140 \mu \mathrm{g} / \mathrm{kg}$ over $3 \mathrm{~min})$ stress perfusion imaging was performed using the same geometry and giving an identical bolus of contrast agent as during rest imaging. Sensitivity, specificity, positive/ negative predictive values, and diagnostic accuracy were calculated according to standard definitions.

Results: A total of 123 vascular territories (left anterior descending artery, left circumflex artery and right coronary artery) were examined. Among them, 11 were excluded due to insufficient image quality. There were 45 coronary arteries stenosis $\geq 70 \%$ by quantitative coronary angiography. Comparisons between $1.5 \mathrm{~T}$ and 3T showed similar efficacy Results: $89 \%$ vs $91 \%$ for sensitivity, $93 \%$ vs $90 \%$ for specificity, $89 \%$ vs $85 \%$ for positive predictive value, $93 \%$ vs $94 \%$ for negative predictive value and $91 \%$ vs $90 \%$ for diagnostic accuracy, respectively.

Conclusion: In this intra-individual comparative study, adenosine-stress perfusion using a gradient-echo sequence at $3 \mathrm{~T}$ is feasible in a clinical routine setting and not inferior to 1.5T SSFP-based adenosine-stress perfusion.

\section{$D-50$}

Is Gd chelates concentration an issue for peripheral MRA diagnostic performance?

S. Haneder, U.I. Attenberger, S.O. Schönberg, H.J. Michaely; Mannheim/DE

Purpose: The purpose of this prospective, single center, intra-individual study is to compare the efficacy of peripheral contrast-enhanced magnetic resonance angiography (CE-MRA) between $0.5 \mathrm{M}$ gadoterate $\left(\right.$ Dotarem $\left.^{\circledR}\right)$ and $1.0 \mathrm{M}$ gadobutrol (Gadovist ${ }^{\circledR}$ ).

Materials and Methods: After IRB approval, 25 consecutive patients with Peripheral Arterial Occlusive Disease (PAOD) stage II or III will be included in this ongoing trial. Within 30 days each patient will obtain two CE-MR exams performed at 3.0T (TimCT Trio, Magnetom, Siemens, Erlangen, Germany). All patients receive $0.1 \mathrm{mmol} / \mathrm{kg}$ BW gadoterate at $1.0 \mathrm{~mL} / \mathrm{sec}$ and $0.1 \mathrm{mmol} / \mathrm{kg} \mathrm{BW}$ gadobutrol at $0.5 \mathrm{~mL} / \mathrm{sec}$ in a double-blinded, randomized manner. The blinded, intra-individual data sets will be independently assessed by two radiologists. For evaluation purpose the vascular tree will be divided in 21 segments. The over all image quality will be rated on a five-point scale. Both readers will rate all segments concerning the assess ability. The grading and the length of all significant stenosis $(>50 \%)$ will be measured. Furthermore the visualization of potential collateral circulation, the diagnostic confidence (five-point scale) and the venous overlap (four-point scale) will be rated. The pedal vessels and smaller branches will be graded for visualization on a 4-point scale in the foot territory. The signal intensity measurements will be performed by a single investigator with both blinded data sets displayed parallel for placement of the regions of interest (ROI). The circular ROIs will be placed on one side of the vascular trunk in the common iliac, the popliteal and the calf artery. Signal to Noise Ratio (SNR) and Contrast to Noise Ratio (CNR) will be computed. Descriptive statistics and statistical tests, including t-tests for comparison purpose will be performed using a $5 \%$ significance level.

Key Words: 3.0T; magnetic resonance angiography; gadoterate; gadobutrol

\section{$D-51$}

Questions and conclusion

C. Ayuso; Barcelona/ES
$12: 30-13: 45$ Room C

organised by GE Healthcare

SY 18

\section{Changing the dose paradigm, enhancing CT diagnosis and optimising patient care}

Moderator:

J. de Mey; Brussels/BE

\section{$D-52$}

Gemstone Spectral Imaging (GSI): A significant advance in the diagnostic performance of $\mathrm{CT}$ as an imaging modality?

M. Bourne; Cardiff/UK

The characterization of soft tissue(s) with conventional CT can be suboptimal, especially for small lesions. Additional diagnostic examinations may be required to verify the diagnosis, adding time and cost to the patient's diagnostic pathway. Gemstone Spectral Imaging (GSI) is a novel, dual energy acquisition that improves the diagnostic power of CT to characterize soft tissue(s). It achieves this by transforming the CT attenuation data into various material densities. The spectral properties of iodine and water can be highlighted for the demonstration of lesion perfusion or to derive virtual non-contrast images from a contrast enhanced examination. The additional information provided by the GSI acquisition facilitates an improved assessment of lesion structure and perfusion. This has lead to a significant advance in the diagnostic power of CT for the characterization of lung nodules, liver lesions and renal stones. Monochromatic $\mathrm{KeV}$ images can be generated from the GSI acquisition. These monochromatic data sets can be utilized to improve image quality by reducing artefacts and to optimize lesion to background contrast to noise. The monochromatic image depicts how the imaged object would look if the X-ray source produced $\mathrm{X}$-ray photons at a single energy. The monochromatic energy is selectable, with higher energies yielding less contrast between materials and yielding more contrast with low energies. In summary, the GSI acquisition provides significant new and unique information for the characterization of soft tissues and improved image quality. This results in a significant improvement in the diagnostic power of CT across a range of applications and clinical scenarios.

\section{$D-53$}

Pediatric low dose CT with GE discovery CT 750HD: Experiences from the Queen Silvia Children's Hospital, Gothenburg, Sweden

F. Stålhammar; Göteborg/SE

Radiation dose is a major concern in computed tomography (CT) imaging, especially for sensitive organs or populations. In one survey, only $43 \%$ of facilities adjusted CT techniques for children, whilst another survey found that $80 \%$ of CT studies in children were not managed by paediatric radiologists. Iterative reconstruction techniques can substantially decrease radiation dose during CT scans. Adaptive Statistical Iterative Reconstruction (ASiR) from GE Healthcare is the first iterative reconstruction method available in routine practice. ASiR reduced the perception of image noise when radiation dose was decreased by as much as $65 \%$. Image noise for low-dose CT ( $50 \%$ of routine dose) with ASiR was almost identical to that obtained by routine-dose CT without ASiR. In our Hospital, ASiR has allowed a radiation dose reduction. This is particularly beneficial for young patients with conditions which cannot be diagnosed conclusively using other techniques. ASiR also benefits patients requiring repeat examinations, as the total radiation dose can be reduced substantially. In our hospital, the main contrast media (CM) used for CT examinations are iodixanol and iohexol. lodixanol is preferred in patients at high risk for contrast-related reactions, such as infants with immature renal function and those at increased risk for adverse events due to prolonged elimination of iodinated CM. lodixanol has been shown to be associated with significantly less nephrotoxicity than a number of LOCM in high-risk patient groups. lodixanol is also preferred for patients undergoing cardiac $\mathrm{CT}$ as the influence of $\mathrm{CM}$ on cardiac function is crucial, and the isosmolality of iodixanol helps minimise cardiac effects.

\section{$D-54$}

Contrast media: How to optimise enhancement and minimise patient risk A. Laghi; Latina/IT

Technological developments have improved the quality of computed tomography (CT) images and the speed with which they are obtained, yet the methods used to optimize images and minimise the risks continue to evolve. When designing protocols for cardiovascular and body CT applications, an understanding of con- 
trast medium (CM) dynamics is essential. lodine flux, injection time, and cardiac output all affect arterial enhancement, whilst iodine dose per unit body weight is regarded as a key determinant of parenchymal enhancement. Cardiac arteries pose particular challenges for CT imaging, due to their small diameter and complex anatomy, combined with the potential for extensive vessel wall disease. Attempts to optimise the diagnostic utility of CTA have increasingly focused on the choice of $\mathrm{CM}$. Whilst a CM with a high iodine concentration might be expected to result in the best enhancement, a "one size fits all" approach may not always produce the best images. Injection of LOCM can be associated with discomfort, heat, pain, and flushing. Osmolality is regarded as the best predictor of cardiac effects. Unlike other $\mathrm{CM}$, iodixanol is isosmolar with blood and contains a physiological ratio of sodium and calcium ions to help reduce the risk for arrhythmias and to prevent contractile failure. Patients who develop contrast-induced nephropathy (CIN) have highe mortality rates than patients who receive $\mathrm{CM}$ without developing $\mathrm{CIN}$. The impact of $\mathrm{CM}$ selection on the incidence of $\mathrm{CIN}$ has been the focus of a number of clinical studies. Among high-risk patient populations, several studies have shown that $\mathrm{CIN}$ is less common with isosmolar iodixanol than with various LOCM.

\section{organised by Bracco}

\section{SY 19}

Programme not available by date of print

\section{organised by GE Healthcare}

\section{SY 20}

\section{Cases of suspected recurrences in oncology: Options and solutions \\ Moderator:}

N.N.

\section{$D-55$}

Clinical benefits of multimodality fusion software for interventional oncology

V. Vilgrain; Clichy/FR

Flat panel detector C-arm angiography system allows not only improvement in fluoroscopic image quality but also new developments. This imaging tool generates three-dimensional (3-D) volumes from data obtained during $\mathrm{C}$-arm rotation such as $3 \mathrm{D}$ angiography and CT like images. 3-D angiography provides an accurate depiction of vessel morphology with low concentrations of contrast media whereas CT like images give information on soft tissues. The volume data set produced by the workstation can be interactively reformatted using maximum intensity projections and multiplanar reconstructions and multimodality fusion can now be done between these volumes and CT or MR images. We have reviewed our 18-month experience performed in a university hospital highly specialised in liver diseases especially in two indications: transarterial chemoembolization (TACE) of liver malignancies and radiofrequency ablation (RFA) of liver tumors. During TACE, 3-D angiography has been shown very interesting in depicting feeding arteries and this technique has altered the catheter position anticipated by attending interventional radiologists in a significant number of cases. At the end of the procedure, fusion between CT like images and preprocedural CT images can assess if the treatment has been made in the entire tumor or if the catheter should be placed in other arteries. During RFA, CT like images can confirm the position of the needles especially when multipolar technique is used. At the end of the procedure, fusion between CT like images and preprocedural CT or MR images can assess if the treated area covers the tumor. Indeed, this new developments increase capabilities for interventional radiology procedures and improve the diagnostic confidence in positioning catheter or needle. Nevertheless, these advantages have to be balanced with some limitations like the learning curve to apply quickly this acquisition and post-process the volumes. Also the image quality and dose tradeoff, compared to conventional CT, have to be considered.

\section{$D-56$}

Innovative $\mathrm{PET} / \mathrm{CT}$ approach in restaging of the non-small cell lung cancer M. Dziuk; Warsaw/PL

The fusion of functional and morphological information by means of PET/CT significantly improves the diagnostic accuracy, resulting from more precise localization and tumor size estimation compared with PET alone. Tumor staging is more accurate with PET/CT than with CT alone or with PET alone. The greatest source of error in accurate localization and quantification on PET or PET/CT in lung cancer are respiratory movements. Motion artifacts influence both PET and CT parts of the study having an even worse impact on combined PET/CT due to aggregating of errors and additional spatial misalignment. Gated PET/CT improves image quality allowing for the achievement of the true resolution of the PET/CT scanner by means of motion detection, characterization and compensation. The discriminative value of gated PET/CT may be especially important in patients with non-small cell lung cancer referred for the assessment of the new lesions near diaphragmatic area such as lung nodule or hilar lymph nodes after the initial treatment or during the follow up. In this clinical setting gated PET/CT, if negative may serve as the modality allowing to exclude or confirm the recurrent disease. Moreover it may be helpful to restage the disease more precisely. This is crucial for treatment planning in patients with recurrent disease.

\section{$D-57$}

Ultrasound fusion imaging for treatment planning and guidance

E. Leen; London/UK

All imaging modalities have their advantages and limitations and the development of fusion imaging has been to strengthen some of the weaknesses of one imaging technique by another in specific clinical fields. Conventional ultrasound imaging is unique in its real time capability but is limited in the detection of focal liver lesions. Whilst CT and MRI are highly sensitive in that respect, they lack the real-time capability. Hence the fusion of US with CT/MRI/CTPET for guidance of interventional procedures such as ablative therapy and biopsies is an obvious step forward. Ultrasound fusion imaging and navigation enables the correct occult small Lesion identified on CT/MR to be characterised on US or contrast enhanced ultrasound and allows therapy planning by targeting identified lesions for future biopsy or RFA. This fusion imaging helps in therapy monitoring by recalling saved markers for the index lesions, in evaluating cancer therapy with US tracking index lesions for size measurement and in assessing antivascular therapies of marked index lesionsf or accurate perfusion plane acquisitions. It also adds great value in treatment guidance (a) by identifying correct lesion to be treated matching with the CT referal scan (b) by targeting Lesion which is seen on CT/MR but not on US - Occult Lesion, (c) by guiding RFA needle during ablation despite the area being obscured with microbubbles and (d) Planning RFA strategy based on CT images.

\section{$D-58$}

The use of a 3.0T MRI equipment in a neurosurgery operating theater D. Netuka; Prague/CZ

The MR suite is adjacent to a neurosurgical OR suite, separated by a sliding RF screen room door that enables surgical patient transfer to the MR while preserving the sterile OR environment. Intraoperative MR imaging is achieved through a specialized patient table docking and transport system, which connects the Maquet surgical table to a specialized Signa MR table. The system maintains consistent patient positioning within the 3.0T compatible MAYFIELD® MR skull clamp between the surgical and MR suites. "By keeping the patient's head fixated within the skull clamp, we can image the patient and accurately upload the data to our neurosurgical system, as well as, quickly fuse the intra-operative with the pre-operative images." The bottom line for implementing an iMRI OR suite is the clinical result. Identifying unexpected tumor remnants that can be removed during the initial surgery, and the ability to confirm that no post-operative complications are present, is the most encouraging news that a surgeon can share with patients and their families.

organised by Philips Healthcare

SY 21

Programme not available by date of print 
organised by Esaote

\section{SY 22}

Diagnosis and therapy in ultrasound: What's next?

\section{Moderators:}

C. Bartolozzi; Pisa/IT

Y. Menu; Paris/FR

\section{$D-59$}

Abdominal ultrasound: Faster and reliable interventional procedures

L. Solbiati; Busto Arsizio/IT

Technical aspects and clinical applications of Fusion Imaging system will be addressed. A new powerful technology capable to offer Virtual Navigation merging real time Ultrasound with pre-acquired multi-modality examinations (CT, MR, PET) will be presented.

\section{D-60}

Breast ultrasound: Extend the vision of daily screening

H. Dobson; Glasgow/UK

A modern vision of the daily routine service on breast screening, based on the available Ultrasound technology will be presented. The role of "high frequency imaging", $3 \mathrm{D}$ acquisition and elastosonography, will be treated, and correctly addressed in the patient clinical management.

\section{D-61}

Vascular ultrasound: Towards preventive healthcare

K.D. Reesink; Maastricht/NL

The technical background of the RF-Based vascular ultrasound system capable of an accurate and reproducible measurements of arterial wall thickness and stiffness will be presented. The clinical advantages of such technical development in terms of a real-time examination and the potential of more precise risk stratification will be addressed.

\section{organised by Toshiba}

\section{SY 23}

Programme not available by date of print 